\title{
Pattern of Uncomplicated Malaria Treatment and Antimalarial Prescription Practices among Health Workers in the Littoral Region of Cameroon: An Assessment of Ten Years Post-Malaria Treatment Policy Change
}

\author{
Thomas Kuete ${ }^{1}$, Emmanuel Essono Mvoa ${ }^{1}$, Jacques Yinyang ${ }^{1}$, Aurelien Epanya Priso ${ }^{1}$, \\ Patrice Cordier Gougue Chamabe', Emmanuel Mpondo Mpondo ${ }^{1}$, Albert Same Ekobo',2 \\ ${ }^{1}$ Faculty of Medicine and Pharmaceutical Sciences, The University of Douala, Douala, Cameroon \\ ${ }^{2}$ National Roll Back Malaria Committee, Yaoundé, Cameroon \\ Email: "thomaskuete@hotmail.com
}

Received 8 April 2016; accepted 20 June 2016; published 23 June 2016

Copyright @ 2016 by authors and Scientific Research Publishing Inc.

This work is licensed under the Creative Commons Attribution International License (CC BY).

http://creativecommons.org/licenses/by/4.0/

cc) (7) Open Access

\section{Abstract}

Following highly prevalent Plasmodium resistant strains to antimalarial monotherapies in malaria endemic countries, uncomplicated malaria treatment policy changed to artemisinine-based combination therapies (ACTs). After adoption of this new treatment policy in a country, sufficient care is needed to be taken to prevent occurrence of resistance to the latest drugs. As Cameroon shifted to ACT in 2004, this study aimed to assess knowledge and practices of health workers in government health facilities of the Littoral region regarding mild malaria management in health facilities as well as according to prescription qualities of ACTs in leaflets received in pharmacies. A total of 66 physicians and 16 nurses were questioned in 10 health facilities and 503 medical leaflets with ACTs prescriptions were viewed in 17 pharmacies. All medical workers questioned correctly were defined mild malaria and were aware of the antimalarial policy change in Cameroon. Overall ACTs prescription for mild malaria management in children and adult patients was $72.2 \%$ and $87.8 \%$ respectively. An important proportion of health workers prescribed antimalarial monotherapies and non recommended antimalarial for uncomplicated malaria treatment. $\mathbf{3 1 . 7 \%}$ of participants did not systematically recommend laboratory diagnostic test before antimalarial prescription. Of leaflets viewed in pharmacies, ACTs were prescribed by physicians, nurses and

\footnotetext{
${ }^{*}$ Corresponding author.
}

How to cite this paper: Kuete, T., et al. (2016) Pattern of Uncomplicated MalariaTreatment and Antimalarial Prescription Practices among Health Workers in theLittoral Region of Cameroon: An Assessment Ten Years Post-MalariaTreatment Policy Change. Pharmacology \& Pharmacy, 7, 217-225. http://dx.doi.org/10.4236/pp.2016.76028 
laboratory technicians. Age was the only criteria for ACTs prescription. Appropriate ACTs quality prescription ranged between $81.2 \%$ and $94.4 \%$. Of the ACTs prescribed, blisters had the highest (92.9\%) appropriate quality prescription and solutions the lowest $(83.3 \%)$. According to qualification of prescribers, physicians had the highest score $(93.1 \%)$ of appropriate quality prescription and laboratory technicians the lowest score $(28.1 \%)$. For all ACTs containing medical leaflets, concomitant medications were recorded namely antipyretic $(\mathbf{7 3 . 9 \% )}$, antibiotic $(\mathbf{2 1 . 9 \% )}$, non steroid anti-inflammatory $(19.9 \%)$ or vitamins $(18.1 \%)$. Data gathered indicated that although health workers were aware of uncomplicated malaria treatment policy change in Cameroon, mild malaria mismanagement was prevailing in health facilities of the Littoral region and ACTs quality prescription in medical leaflets was not optimal. Therefore, awareness is still needed among prescribers in order to prevent or at least slow the occurrence of Plasmodium resistant strains to ACTs in Cameroon.

\section{Keywords}

Uncomplicated Malaria, Knowledge, Treatment, Antimalarial Prescriptions, Quality, Artemisinin-Based Combination Therapies

\section{Introduction}

Malaria is a life threatening parasitic disease leading to an estimate 584,000 deaths in 2013, the WHO African Region owing the heaviest burden with an estimated $90 \%$ of all malaria deaths worldwide, and children aged under 5 years old accounting for $78 \%$ of all deaths [1] [2]. The disease is due to erythrocyte inhabiting parasites of the genus Plasmodium, and infected subjects may develop different clinical stages ranging from asymptomatic, to uncomplicated or/and finally to severe malaria. The disease is mostly endemic in Africa, America and Asia where an estimated 3.2 billion people are at risk of infection, and 1.2 billion are at high risk of getting malaria in a year [1].

Since the early 1990s, the occurrence of Plasmodium chemoresistant strains to the cheapest and widely available antimalarial monotherapies becomes a major obstacle to treatment of malaria in endemic countries. Plasmodium chemoresistant strains occurred to almost all monotherapeutic antimalarial drugs [3] [4] in Thailand in 1957 then increasingly spread from East to West, towards South-Eastern Asia and reached sub-Saharan African countries in the years 1970s [4] [5]. This outbreak of Plasmodium chemoresistant to monotherapies contributed to a worldwide increase in malaria-related mortality, particularly dramatic in sub-Saharan Africa as few affordable alternatives were available. Drug pressure was identified as one of the key factors for the emergence and spread of resistance through the extensive use and misuse of antimalarial drugs. Incorrect uses of drugs probably lead to the selection of resistant parasites which became particularly evident during the Global Malaria Eradication campaign, launched by World Health Organization (WHO) in 1955.

Regarding the rapid widespread of this chemoresistance in malaria endemic countries, medical experts joined to WHO and recommended in 2001 the implementation of artemisinine-based combinations therapies (ACTs) as first line treatment for a better management of uncomplicated malaria in infected patients as a radical replacement of monotherapies [6]. Since then, antimalarial monotherapies were no more recommended for first line treatment of uncomplicated malaria in malaria endemic countries with high chemoresistance, and ACTs became the main hope for current uncomplicated malaria treatment because of their effectiveness for the treatment of multidrug-resistant $P$. falciparum infections. ACTs was demonstrated in many studies to have higher and sustainable parasitological cure rates over monotherapies thus reducing therapeutic failure rates including $P$. falciparum resistant strains in sub-Sahara Africa malaria endemic countries compared to monotherapies [7]-[10]. Furthermore, ACTs have demonstrated effective on gametocytes thus limiting much more transmission [11][13]. The T3 initiative: Test-Treat-Track was another step forward WHO guidelines on malaria management set in order to prevent another occurrence of chemoresistance to ACTs. The T3 initiative recommends for suspected malaria case, a laboratory confirmation prior to specific treatment; then a follow-up of treated subject should be 
implemented until cure [14]. Thus a key success of the new treatment policy depends on the adherence of health workers to the guidelines followed by the compliance of patients with the recommended treatment schemes.

Correct implementation of the new treatment policy may not be rapidly and well translated in health workers if they are not well informed or they do not comply with the new treatment recommendations. Quality prescriptions by health workers is therefore a main key factor since its influence the outcomes of resistant strains control therefore influencing the national public health disease control policy.

Cameroon switched to ACTs for first line treatment of mild malaria in 2004 and selected up-to-date the following therapeutic options: artesunate-amodiaquine combination (AS + AQ), artemether-lumefantrine (AL), artesunate-mefloquine (AS + MQ), dihydroartemisnine-piperaquine phosphate (DHA + PP), dihydroartemisinine-piperaquine phosphate-trimethoprime (DHA + PP + Trim) [15]-[18]. Quinine, intramuscular use of artemether or intravascular use of artesunate was recommended only for management of severe malaria in Cameroon [6] [15].

This cross-sectional study aimed to investigate knowledge and practices of prescribers concerning management of mild malaria in government health facilities, and treatment practices with special reference to ACTs prescription in leaflets received in recommended pharmacies in the Littoral region of Cameroon 10 years after the country opted to ACTs for first line treatment of mild malaria.

\section{Material and Methods}

\subsection{Study Period and Place}

This was a cross-sectional study undergone from 2013 to 2014 in 10 health facilities selected in the four main divisions of the Littoral region in Cameroon, and in 17 pharmacies situated in the 5 subdivisions of Douala town.

Health facilities used in this study were the 2 reference hospitals of the Littoral region namely the Douala General Hospital and the Douala Laquintinie Hospital, the 2 regional hospitals of the Littoral region namely Edea and Nkongsamba regional hospitals, 3 district hospitals selected in three administrative divisions and 3 government integrated health centres also selected in three administrative divisions.

The healthcare system in Cameroon is classified as follow according to healthcare performances: reference hospitals, central hospitals and university teaching hospitals lead the healthcare system and are thought to provide high quality healthcare. They are followed by regional hospitals which like the preceding provide high quality healthcare but are often less equipped, unlike reference hospitals have less healthcare specialities and less specialist physicians. Regional hospitals are followed by health district with also high healthcare capacities. Each health has a district hospital which leads and supervises activities of many governmental integrated health centres, private hospitals and clinics.

\subsection{Ethics}

The study protocol was approved by the Littoral Regional Delegation of public health and the ethic committee of the University of Douala. A health facility or pharmacy was included in the study only after obtaining a written authorization from its director. Therefore, an authorization was sought from the directors of the health facilities and pharmacies through a letter for authorization to undergo the study in the facility sent by the study investigators. For data collection from medical staff or those presenting leaflets in pharmacies, the protocol was explained to them then they were asked for their participation in the study.

\subsection{Study Criteria}

All permanent medical workers namely physician or nurse encountered in any of the selected health facilities who gave its consent was included in the study. In pharmacies, all ACT-containing medical leaflet presented by any patient or relative was included in the study. The patient or relative should have given its consent before inclusion in the study, thus any subject who refused its consent was not allowed in the study.

Choice of health facilities was made among the three categories of national health stratification namely reference hospitals (RH), district hospitals (DH) and integrated health centres (IHC). For pharmacies, only recommended pharmacies were selected. Hospital pharmacies and road vendors' pharmacies were also excluded from the study. 


\subsection{Data Collection}

Data collection in health facilities consisted in a questionnaire to be filled by the health worker. This questionnaire sought the name of the health facility and for each participant, its medical qualification, any diagnostic test before anti-malarial drug prescription (health professional presumptive diagnosis or laboratory-confirmed, type of anti-malarial often recommended to patient suffering from uncomplicated malaria, knowledge about the criteria used for dosage (weight or height) for any of the anti-malarial prescribed.

\subsubsection{Management Practices Vis-A-Vis Mild Malaria}

Data concerning knowledge and practices of prescribers upon management of mild malaria were collected through a tested questionnaire seeking: a) any diagnostic test before anti-malarial prescription; b) anti-malarial types prescribed for management of mild malaria.

\subsubsection{Quality Prescriptions of Acts in Medical Leaflets}

Data collected from ACTs-containing medical leaflets in pharmacies included age and weight of the patient, the name of ACT prescribed, appropriateness of the treatment dosages namely quantity at each intake and duration of treatment according to manufacturer and the national Public Health ministry guidelines, concomitant medications to the ACT regimen prescribed in the leaflet, name and qualification of the prescriber.

Data were statistically analyzed using Chi square test and difference was considered significant for $\mathrm{p}<5 \%$.

\section{Results}

As indicated in Figure 1, a total 82 medical workers were questioned in 10 health facilities. Also, a total of 503 medical leaflets with ACTs prescriptions were viewed in 17 pharmacies situated in the 5 subdivisions of Douala town.

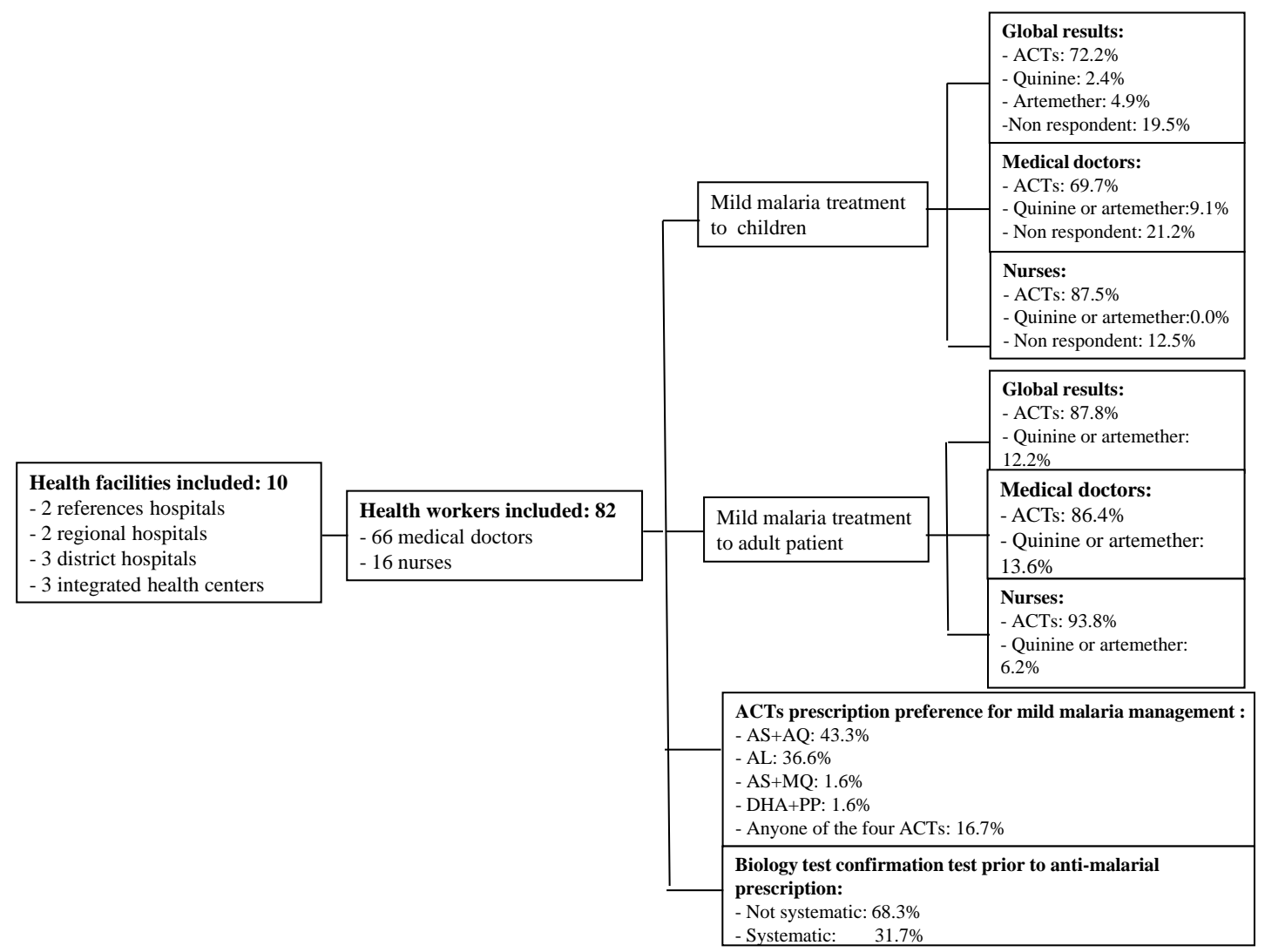

Figure 1. Uncomplicated malaria treatment knowledge and practices in 10 health facilities of the Littoral region in Cameroon. 


\subsection{Knowledge and Practices about Mild Malaria Treatment in Health Facilities}

Participants included $80.5 \%$ medical doctors and 19.5\% nurses. Health facilities in which participants were recruited included 2 reference hospitals, 2 regional hospitals, 3 districts hospitals and 3 integrated health centres. According to data from census, these health facilities had a total of 228 physicians and 529 nurses. $48.5 \%$ of medical workers encountered participated in the study.

All medical workers who participated in the study correctly defined mild malaria as feverish patient with or without any symptom in absence of any sign of complicated malaria as defined in WHO criteria. Also, all participants reported having already heard about ACT as the only recommended anti-malarial medications for mild malaria treatment in Cameroon.

\subsubsection{Malaria Prescription Trends for Mild Malaria Treatment in Children}

As shown in Figure 1, 72.2\% participants reported using an ACT for treatment of mild malaria whereas 2.4\% and $4.9 \%$ reported using quinine or artemether respectively. $19.5 \%$ of the participants did not give any answer to this question.

According to qualification of prescribers, 69.7\% of medical doctors reported prescribing an ACT for mild malaria management in children, $21.2 \%$ of the physicians gave no answer to this question, and $9.1 \%$ prescribed either quinine or artemether. Among nurses, 87.5\% reported prescribing an ACT, none prescribed quinine or artemether, and $12.5 \%$ did not answer this question.

\subsubsection{Malaria Prescription Trends for Mild Malaria Treatment in Adult Patients}

As indicated in Figure 1, 87.8\% of medical workers reported prescribing an ACT for mild malaria management in adult subjects whereas $12.2 \%$ reported prescribing quinine or artemether. An ACT was prescribed by $86.4 \%$ of physicians and $93.8 \%$ of nurses. $13.6 \%$ of medical doctors and $6.2 \%$ of nurses reported prescribing quinine or artemether for treatment of malaria in adult patients.

The right ACT dosage according either to age or weight was appropriate in $61.7 \%$ cases whereas $38.3 \%$ of health workers did not answer. Correct treatment duration was given by $83.3 \%$ of participants and $16.7 \%$ of them gave no answer.

Globally, artesunate-amodiaquine combination was the most frequently prescribed ACT (43.3\%) followed by artemether-lumefantrine combination (36.6\%). Artesunate-mefloquine or dihydroartemisinine piperaquine phosphate combinations were the least recommended by health workers and $16.7 \%$ of participants reported prescribing anyone of the ACT listed.

\subsubsection{Laboratory Diagnostic Tests before Treatment Prescription}

68.3\% reported not systematically recommend laboratory results prior to anti-malarial prescription. Among anti-malarial prescribers who systematically prescribed anti-malarial after laboratory results, three mains tests were recorded namely thick blood smear, thin blood smear and malaria rapid diagnostic test (RDT). Thick blood smear was the most frequently recommended by health workers (51.2\%), followed by RDT (45.1\%) and thin blood smear (4.9\%).

\subsection{ACTs Quality Prescriptions in Medical Leaflets Received in Pharmacies}

As shown in Figure 2 below, a total 503 medical leaflets with ACTs prescriptions were viewed in 17 pharmacies selected in the 5 subdivisions of Douala town. Mean age of patients with an ACT prescription was $18.5 \pm$ 15.5 years (range: 21 days to 69 years). Patients aged at least 14 years and under five years subjects represented $45.5 \%$ and $17.3 \%$ of the sampled leaflets respectively.

Prescribers were predominantly medical doctors (83.1\%). Other ACT prescriptions were given by nurses (10.5\%) and laboratory technicians (6.4\%).

Five main ACTs as common international denomination (CID) were recorded in leaflets namely Artemetherlumefantrine (AL), Dihydroartemisinine-piperaquine phosphate (DHA + PP), Artesunate-amodiaquine (ASAQ), Artesunate-mefloquine (AS + MQ), Artesunate-sulfadoxine-pyrimethamine (AS + SP) and Dihydroartemisinine-piperaquine phosphate-trimethoprime (DHA + PP + Trim).

Three main ACTs presentations were prescribed in leaflets namely blisters or tablets, oral solution and sachets. Tablets forms were most represented (59.8\%) and sachets the least frequent form in leaflets (3.6\%). Oral solu- 
tion and water soluble forms were found in $28.6 \%$ and $8.0 \%$ leaflets respectively.

Adequate ACT quality prescription was recorded in $88.3 \%$ medical leaflets and inadequate in $11.7 \%$ medical leaflets.

ACTs quality prescriptions varied significantly according to qualification of prescribers $(\chi 2=121.2$; df2; $\mathrm{p}<$ 0.05). Medical doctors had the highest score of adequate ACTs prescriptions (93.1\%), whereas laboratory technicians had the lowest score (28.1\%). ACTs prescriptions by nurses were adapted in $86.8 \%$ leaflets.

As shown in Figure 2, quality of ACTs prescriptions was not statistically different with respect to ACTs $(\chi 2=$ 5.1; df5; $p>0.05$ ). Appropriate prescriptions were over $80 \%$ irrespective of ACT, mean score of ACTs appropriate prescription recorded in leaflets was $83.3 \%$. Scores of prescriptions quality of recommended ACTs ranged between $81.2 \%$ for ASAQ and $94.4 \%$ for DHA + PQ + Trimetoprime.

As shown in Figure 2, quality prescription was also significantly influenced by the ACTs presentation $(\chi 2=$ 11.5; df3; $\mathrm{p}=0.009)$. Oral solution presentation had the lowest prescription quality score (83.3\%) and blisters the form the highest score (92.9\%). Sachets forms appropriate prescriptions score in leaflets was $88.9 \%$.

Investigation of prescriptions security indicated that all leaflets had the frequency of treatment but none of indicated the exact hour of the day at which the treatment should be taken by the patient. Age or weight of patients was not mentioned in $91.9 \%$ and $97.4 \%$ of leaflets respectively.

\subsection{Concomitant Medications to ACT Prescriptions}

In all leaflets received in pharmacies, ACTs were co-prescribed with at least another but non anti-malarial medication. The most commonly prescribed concomitant medication was an antipyretic alone in $34.4 \%$ leaflets, an antibiotic in $21.9 \%$ cases, a non steroid anti-inflammatory $19.8 \%$ leaflets, vitamins in $18.1 \%$ cases. Co-prescription with an antipyretic plus either an antibiotic or non steroid anti-inflammatory or vitamin or combination of two or three of the medications was recorded in $73.9 \%$ of medical leaflets. None of the associated medication was known to interact with the ACTs prescribed in the corresponding leaflet. Over 14 years old patients were less likely significantly to have an antibiotic and vitamins co-prescription than younger ones.

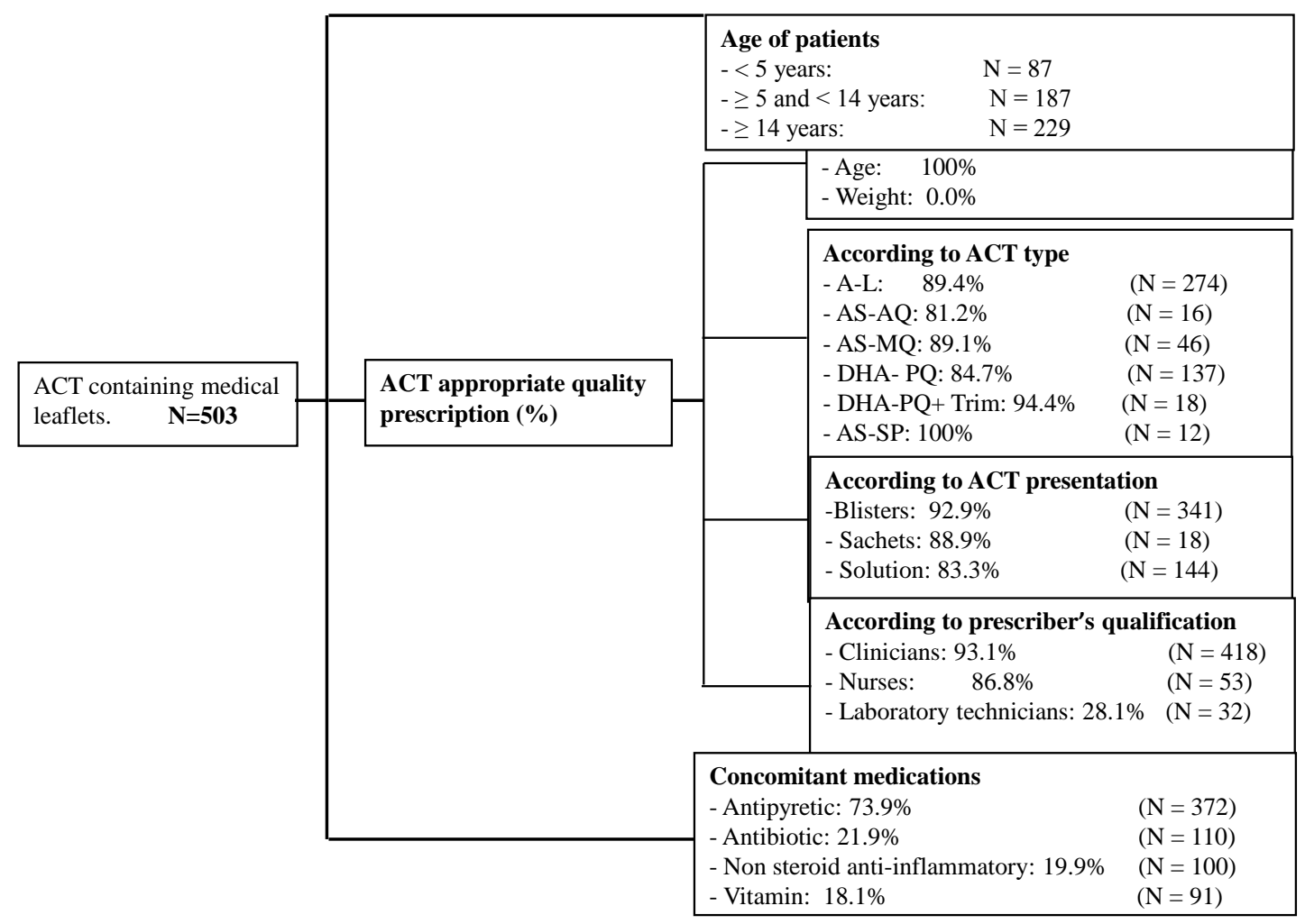

Figure 2. Flow chart of ACTs quality prescriptions in leaflets received in pharmacies in Douala town, Cameroon. $\mathrm{N}=$ Sample size. 


\section{Discussion}

This study has underlined the knowledge level, practices and quality prescriptions of health practitioners upon treatment of mild malaria in Douala town a decade after changing of therapeutic guidelines towards mild malaria by the national health ministry.

Although the proportion of health workers in the health facilities who were aware of the ACTs exclusive use for uncomplicated malaria management was high, there still a need for more sensitization in health facilities to achieve more adhesion of health practitioners. In the questionnaire filled by health workers in health facilities, some of them still prescribe anti-malarial monotherapies for uncomplicated malaria management. Prescription of the latest anti-malarial monotherapies namely quinine, intravenous artemether or intramuscular artesunate all designed only for severe malaria case treatment may either signify non-acceptance of the changed drug policy or lack of awareness though many training campaigns have been undergone in almost health facilities throughout the national territory. Health practitioners in health facilities reported most commonly recommending ACTs for mild malaria treatment therefore respecting the national and WHO guidelines on uncomplicated malaria management. However, inadequate prescriptions were common in leaflets received in pharmacies as well as in answers to questionnaires recorded among health workers in health facilities. The occurrence of such inadequate prescriptions influence treatment responses and may progressively contribute to outbreak of less responsive Plasmodium parasites to ACT treatment and later to chemo resistant strains. Concomitant medications alongside ACTs prescriptions may be explained either the desire of the prescriber to rapidly clear most symptoms felt by the patients or the by insistence of patients on receiving these concomitant drugs to feel better.

As a limitation of this study, the reasons of persistent prescription of these anti-malarial monotherapies for management of mild malaria in health facilities in the Littoral region of Cameroon were not investigated. Since the inappropriate use of anti-malarial monotherapies in the past was pointed as a key which contributed to decreased drug sensitivities, inappropriate anti-malarial prescriptions need to be completely banned in health facilities and adequate prescription of the right drug for management of mild malaria as indicated in the national guidelines be promoted [15]. Adhesion of all health workers is urgently needed as recent studies indicated occurrence of artemisinine and derivatives $P$. falciparum resistant strains in Southeast Asia notably in Thailand, Cambodia and Myanmar [19]-[21]. Irrational use of artemisinine and its derivatives as monotherapy could negate one of the goals of combination therapy which is to prevent the emergence of drug resistant malaria parasites.

Another interesting finding was prescription in leaflets received in pharmacies of an ACT not recommended in the country national guidelines namely Artesunate-sulfadoxine-pyrimethamine combination. In the national guidelines for malaria management, sulfadoxine-pyrimethamine (sp) is recommended by the Ministry of Public health of Cameroon only for intermittent preventive treatment in pregnant women [15]. Prescription of artesunate-sulfadoxine-pyrimethamine combination for management of mild was therefore inappropriate.

Management of mild malaria need also be appropriate through better adhesion to the T3 initiative guidelines thus limiting presumptive treatment. However, systematic laboratory test for Plasmodium infection confirmation was not often a rule for all questioned health workers, therefore not respecting the T3 initiative guidelines. In fact, WHO recommends that all persons of all ages in all epidemiological settings with suspected malaria should receive a parasitological confirmation of diagnosis by either microscopy or rapid diagnostic test (RDT), and that uncomplicated $P$. falciparum malaria should be treated with a recommended ACT [6]. Diagnostic testing for malaria is the cornerstone of WHO initiative-T3: Test. Treat. Track-whereby testing of every suspected malaria case ensures appropriate antimalarial treatment and improves malaria surveillance. An awareness amongst the health practitioners in terms of conducting campaigns for effective uncomplicated malaria case management through limitation if not banning of presumptive treatment will certainly contribute to prevent or at least slow down the occurrence of artemisinine-resistant Plasmodium strains in malaria endemic areas.

Despite the fact that ACTs was recommended by an important proportion of health workers questioned in health facilities, inadequate prescriptions are frequent in medical leaflets received in pharmacies. An important proportion of prescribers were unable to answer the questions regarding the drug to be used and dosages for treatment mild malaria treatment. Such unanswered questions may either be related to ignorance or negligence therefore leading to misinterpretation of the related results. The authors of this study suggest that a periodical monitoring of ACTs quality prescriptions is still needed in health facilities for improvement towards high standard ACT prescribing by health workers. 


\section{Conclusion}

The study revealed a persisting prevailing mismanagement of uncomplicated malaria by an important proportion of health workers in the Littoral region of Cameroon despite of availability of national and WHO evide- ncedbased guidelines for uncomplicated malaria treatment. Such mismanagement which included persisting prescription of non-recommended anti-malarial drugs, inappropriate dosages of ACTs in leaflets and persisting presumptive malaria treatment called for awareness among the physicians and other health personnel for better management of mild malaria according to national rules through training to prevent or at least slow the occurrence of Plasmodium resistant strains to artemisinine and derivatives in Cameroon.

\section{Acknowledgements}

This study received no financial support. The authors are grateful to the health facilities medical staff of and to pharmacists who contributed to achieve our study objectives.

\section{Competing Interests}

The authors declare that there are no competing interests concerning this study.

\section{Author's Contributions}

TK, EMM, ASE designed the study. TK, EEM, JY, AEP, PCGC, performed data collection. TK, EMM, ASE: supervised data collection and contributed to writing the paper; TK drafted the paper. All the authors read and approved the final manuscript.

\section{References}

[1] World Health Organization (2014) The World Malaria Report 2014. World Health Organization, Geneva, WHO/HTM/ GMP/2014, 242 p.

[2] World Health Organization (2013) The World Malaria Report 2013. World Health Organization, Geneva, WHO/HTM/ GMP/2013, 286 p.

[3] Flegg, J.A., Metcalf, C.J.E., Gharbi, M., Venkatesan, M., et al. (2013) Trends in Antimalarial Drug Use in Africa. The American Journal of Tropical Medicine and Hygiene, 89, 857-865. http://dx.doi.org/10.4269/ajtmh.13-0129

[4] Packard, R.M. (2014) The Origins of Antimalarial-Drug Resistance. The New England Journal of Medicine, 371, 397-399. http://dx.doi.org/10.1056/NEJMp1403340

[5] D'Alessandro, U. and Buttiëns, H. (2001) History and Importance of Antimalarial Drug Resistance. Tropical Medicine \& International Health, 6, 845-848. http://dx.doi.org/10.1046/j.1365-3156.2001.00819.x

[6] World Health Organization (2001) Antimalarial Drug Combination Therapy: Report of a WHO Technical Consultation. WHO/CDS/RBM, 35.

[7] Sinclair, D., Zani, B., Donegan, S., Olliaro, P. and Garner, P. (2009) Artemisinine-Based Combination Therapy for Treating Uncomplicated Malaria (Review). Cochrane Reviews, 4, 270 p.

[8] Adjuik, M., Agnamey, P., Babiker, A., Borrmann, S., Brasseur, P., et al. (2002) Amodiaquine-Artesunate versus Amodiaquine for Uncomplicated Plasmodium falciparum Malaria in African Children: A Randomised, Multicentre Trial. Lancet, 359, 1365-1372. http://dx.doi.org/10.1016/S0140-6736(02)08348-4

[9] Mutabingwa, T.K., Heller, A., Hallett, R., Ahmed, J., et al. (2005) Amodiaquine Alone, Amodiaquine/Sulfadox- inePyrimethamine, Amodiaquine/Artesunate, and Artemether/Lumefantrine for Outpatient Treatment of Malaria in Tanzanian Children: A Four-Arm-Randomised Effectiveness Trial. Lancet, 365, 1474-1480. http://dx.doi.org/10.1016/S0140-6736(05)66417-3

[10] Ogbonna, A. and Uneke, C.J. (2008) Artemisinin-Based Combination Therapy for Uncomplicated Malaria in Sub-Saharan Africa: The Efficacy, Safety, Resistance and Policy Implementation since Abuja 2000. Transactions of the Royal Society of Tropical Medicine and Hygiene, 102, 621-627. http://dx.doi.org/10.1016/j.trstmh.2008.03.024

[11] Massougbodji, A., Kone, M., Kinde-Gazar, D., Same-Ekobo, A., et al. (2002) Randomised, Doubled-Blind Study on the Efficacy and Safety of Artesunate and Mefloquine (Artequin ${ }^{\circledR}$ ) Given Simultaneously for 3 Days Compared to a Sequential Treatment in Uncomplicated Plasmodium falciparum Malaria in Africa. Transactions of the Royal Society of Tropical Medicine and Hygiene, 96, 655-659. http://dx.doi.org/10.1016/S0035-9203(02)90344-5

[12] Ndiaye, J.L., Faye, B., Diouf, A.M., Kuété, T., et al. (2008) Randomized, Comparative Study of the Efficacy and 
Safety of Artesunate plus Amodiaquine, Administered as a Single Daily Intake versus Two Daily Intakes in the Treatment of Uncomplicated Falciparum Malaria. Malaria Journal, 7, 16. http://dx.doi.org/10.1186/1475-2875-7-16

[13] Ndiaye, J.L., Randrianariveloosia, M., Sagara, I., Brasseur, P., et al. (2008) Randomized, Multicentre Assessment of the Efficacy and Safety of ASAQ-a Fixed-Dose Artesunate-Amodiaquine Combination Therapy in the Treatment of Uncomplicated Plasmodium falciparum Malaria. Malaria Journal, 8, 125. http://dx.doi.org/10.1186/1475-2875-8-125

[14] WHO (2010) Guidelines for the Treatment of Malaria. 2nd Edition, World Health Organization, Geneva. http://whqlibdoc.who.int/publications/2010/9789241547925_eng.pdf

[15] National Malaria Control Programme (2007) Directorates for Management and Use of Artemisinine-Based Combination Therapies (ACT) in Cameroon. Ministry of Public Health Report, 22 p.

[16] Brasseur, P., Agnamey, P., Same-Ekobo, A., Samba, G., et al. (1995) Sensitivity of Plasmodium falciparum to Amodiaquine and Chloroquine in Central Africa: A Comparative Study in Vivo and in Vitro. Transactions of the Royal Society of Tropical Medicine and Hygiene, 89, 528-530. http://dx.doi.org/10.1016/0035-9203(95)90096-9

[17] Ringwald, P., Keundjian, A., Same-Ekobo, A. and Basco, L.K. (2000) Chemoresistance of Plasmodium falciparum in the Urban Region of Yaoundé, Cameroon. Part 2: Evaluation of the Efficacy of Amodiaquine and SulfadoxinePyrimethamine Combination in the Treatment of Uncomplicated Plasmodium falciparum Malaria in Yaoundé, Cameroon. Tropical Medicine \& International Health, 5, 620-627. [Article in French]

[18] Basco, L.K., Same-Ekobo, A., Ngane, V.F., Ndounga, M., et al. (2002) Therapeutic Efficacy of SulfadoxinePyrimethamine, Amodiaquine and the Sulfadoxine-Pyrimethamine-Amodiaquine Combination against Uncomplicated Plasmodium falciparum Malaria in Young Children in Cameroon. Bulletin of the World Health Organization, 80, 538-545.

[19] Tun, K.M., Imwong, M., Lwin, K.M., Win, A.A., Hlaing, T.M., et al. (2015) Spread of Artemisinin-Resistant Plasmodium falciparum in Myanmar: A Cross-Sectional Survey of the K13 Molecular Marker. The Lancet Infectious Diseases, 15, 415-421. http://dx.doi.org/10.1016/S1473-3099(15)70032-0

[20] World Health Organization (2014) Status Report on Artemisinin Resistance. World Health Organization, Geneva.

[21] Spring, M.D., Lin, J.T., Manning, J.E., Vanachayangkul, P., Somethy, S., et al. (2015) Dihydroartemisinin-Piperaquine Failure Associated with a Triple Mutant Including kelch13 C580Y in Cambodia: An Observational Cohort Study. The Lancet Infectious Diseases, 15, 683-691.

\section{Submit or recommend next manuscript to SCIRP and we will provide best service for you:}

Accepting pre-submission inquiries through Email, Facebook, Linkedin, Twitter, etc

A wide selection of journals (inclusive of 9 subjects, more than 200 journals)

Providing a 24-hour high-quality service

User-friendly online submission system

Fair and swift peer-review system

Efficient typesetting and proofreading procedure

Display of the result of downloads and visits, as well as the number of cited articles

Maximum dissemination of your research work

Submit your manuscript at: http://papersubmission.scirp.org/ 